\title{
ON CONNECTIVITY IN MATROIDS AND GRAPHS
}

BY

JAMES G. OXLEY

\begin{abstract}
In this paper we derive several results for connected matroids and use these to obtain new results for 2-connected graphs. In particular, we generalize work of Murty and Seymour on the number of two-element cocircuits in a minimally connected matroid, and work of Dirac, Plummer and Mader on the number of vertices of degree two in a minimally 2-connected graph. We also solve a problem of Murty by giving a straightforward but useful characterization of minimally connected matroids. The final part of the paper gives a matroid generalization of Dirac and Plummer's result that every minimally 2-connected graph is 3-colourable.
\end{abstract}

1. Introduction. The structure of minimally 2-connected graphs was determined independently by Dirac [5] and Plummer [16]. Their work led Murty [10] to examine minimally 2-connected matroids and some of the latter's results were generalized by Seymour [17], [18]. In $\$ 2$ of this paper, we strengthen one such result of Seymour by showing that if $C$ is a circuit in a 2-connected matroid $M$ and for all $x$ in $C$, the restriction $M \backslash x$ is not 2-connected, then provided $|E(M)| \geqslant 4, M$ has at least two disjoint cocircuits of size two contained in $C$. Several corollaries of this theorem are proved and the theorem is also used to derive the corresponding result for a 2-connected graph $G$, the conclusion in this case being that the circuit $C$ meets at least two nonadjacent vertices of $G$ of degree two. This result, which extends a result of Dirac [5] and Plummer [16] for minimally 2-connected graphs, is a strengthening in the case $n=2$ of a result of Mader [8] for $n$-connected graphs. It has a number of corollaries including a new lower bound on the number of vertices of degree two in a minimally 2 -connected graph. Some similar results for minimally $n$-connected graphs and matroids are also obtained for $n \geqslant 3$.

In [10], Murty asks for a characterization of minimally 2-connected matroids. In §3, we give such a result, showing that unless every element of a minimally 2-connected matroid $M$ is in a cocircuit of size two, $M$ can be obtained from two minimally 2-connected matroids on fewer elements by a join operation which is closely related to series connection. This characterization, which is not difficult to prove, is used to give short proofs of the main results of [10].

Dirac [5] and Plummer [16] have shown that a minimally 2-connected graph is 3-colourable and Dirac's argument [5, p. 215] can be extended to show that a minimally $n$-connected graph is $(n+1)$-colourable (see, for example, [1, Corollary 4.7]). In $\S 4$, by generalizing this argument, we establish the corresponding result for minimally $n$-connected matroids when $n=2$ or 3 .

Received by the editors October 19, 1979.

1980 Mathematics Subject Classification. Primary 05B35, 05C40, 05C15; Secondary $05 C 35$. 
The terminology used here for matroids and graphs will in general follow Welsh [23] and Bondy and Murty [2] respectively. Most of the known graph connectivity results to which we shall refer can be found in Bollobás [1, Chapter 1]. The ground set of a matroid $M$ will be denoted by $E(M)$ and, if $T \subseteq E(M)$, we denote the rank of $T$ by rk $T$. We shall write rk $M$ for $\operatorname{rk}(E(M))$ and cork $M$ for the rank of the dual matroid $M^{*}$ of $M$. The restriction of $M$ to $E(M) \backslash T$ will sometimes be denoted by $M \backslash T$ or, if $T=\left\{x_{1}, x_{2}, \ldots, x_{m}\right\}$, by $M \backslash x_{1}, x_{2}, \ldots, x_{m}$. Likewise, the contraction of $M$ to $E(M) \backslash T$ will sometimes be written as $M / T$ or $M / x_{1}, x_{2}, \ldots, x_{m}$. A cocircuit of $M$ having $k$ elements will be called a $k$-cocircuit, and a $k$-element circuit, a $k$-circuit. If $x, y \in E(M)$ and $\{x, y\}$ is a 2-cocircuit, we say that $x$ and $y$ are in series; if instead $\{x, y\}$ is a 2-circuit, then $x$ and $y$ are in parallel. A series class of $M$ is a maximal subset $A$ of $E(M)$ such that if $a$ and $b$ are distinct elements of $A$, then $a$ and $b$ are in series. Parallel classes are defined analogously. We call a series or parallel class nontrivial if it contains at least two elements.

Familiarity will be assumed with the concept of $n$-connection for graphs as defined, for example, in [2, p. 42]. We now recall the definition of $n$-connection for matroids [20]. If $k$ is a positive integer, the matroid $M$ is $k$-separated if there is a subset $T$ of $E(M)$ such that $|T| \geqslant k,|E(M) \backslash T| \geqslant k$ and

$$
\text { rk } T+\operatorname{rk}(E(M) \backslash T)-\operatorname{rk} M=k-1 .
$$

If there is a least positive integer $j$ such that $M$ is $j$-separated, it is called the connectivity $\kappa(M)$ of $M$. If there is no such integer, we say that $\kappa(M)=\infty$. The matroid $M$ is said to be $n$-connected for any positive integer $n$ such that $n<\kappa(M)$. It is routine to show $[20,(12)]$ that

$$
\kappa(M)=\kappa\left(M^{*}\right) .
$$

The familiar notion of connectivity or nonseparability of matroids is related to $n$-connectedness as follows.

(1.2) A matroid is connected if and only if it is 2-connected.

A matroid or graph $H$ is minimally $n$-connected if $H$ is $n$-connected and, for all elements $e$ of $E(H), H \backslash e$ is not $n$-connected.

The notions of $n$-connectedness of a graph $G$ and $n$-connectedness of its cycle matroid $M(G)$ do not, in general, coincide (see [6], [21], [23]). However, [21, pp. $1-2]$

(1.3) if $G$ has no loops and at least three vertices, then $G$ is 2-connected if and only if $M(G)$ is 2-connected; and

(1.4) if $G$ is simple and has at least four vertices, then $G$ is 3-connected if and only if $M(G)$ is 3-connected.

If $G$ is a 2-connected graph without loops, then clearly the set of all edges incident with a vertex of $G$ is a cocircuit of $M(G)$. Such a cocircuit will be called a vertex cocircuit of $M(G)$.

2. Connectivity results for matroids and graphs. The following basic lemmas will be used frequently throughout this paper.

(2.1) LEMMA [20, (6.5)]. If $M$ is a 2-connected matroid and $e \in E(M)$, then either $M \backslash e$ or $M / e$ is 2-connected. 
(2.2) LeMMA [15, LemMa 2.1]. Let $M$ be a matroid and $n$ be an integer exceeding one. Suppose that $e$ is an element of $M$ for which $M / e$ is $n$-connected but $M$ is not. Then either $e$ is a loop of $M$ or $M$ has a cocircuit containing $e$ and having fewer than $n$ elements.

In this section we shall give two extensions of a result of Seymour [18, (2.3)]. The next lemma is the first of these.

(2.3) LeMmA. Let $M$ be a 2-connected matroid having at least two elements and $\left\{x_{1}, x_{2}, \ldots, x_{m}\right\}$ be a circuit of $M$ such that $M \backslash x_{i}$ is not 2-connected for all $i$ in $\{1,2, \ldots, m-1\}$. Then $\left\{x_{1}, x_{2}, \ldots, x_{m-1}\right\}$ contains a 2-cocircuit of $\boldsymbol{M}$.

Proof. We argue by induction on $m$. If $m=1$ or 2 , the result is vacuously true, so suppose $m>2$. As $M \backslash x_{1}$ is not 2-connected, it follows by Lemma 2.1 that $M / x_{1}$ is 2-connected. Moreover, $\left\{x_{2}, x_{3}, \ldots, x_{m}\right\}$ is a circuit of $M / x_{1}$. Thus, if for all $i$ in $\{2,3, \ldots, m-1\}, M / x_{1} \backslash x_{i}$ is not 2-connected, then, by the induction assumption, $\left\{x_{2}, x_{3}, \ldots, x_{m-1}\right\}$ contains a 2-cocircuit of $M / x_{1}$, and hence, as required, $\left\{x_{1}, x_{2}, x_{3}, \ldots, x_{m-1}\right\}$ contains a 2-cocircuit of $M$. We may therefore suppose that for some $i$ in $\{2,3, \ldots, m-1\}, M / x_{1} \backslash x_{i}$ is 2-connected. Thus, as $M \backslash x_{i}$ is not 2-connected, by Lemma 2.2, $x_{1}$ is a loop or a coloop of $M \backslash x_{i}$ and so $\left\{x_{1}, x_{i}\right\}$ is a 2 -cocircuit of $M$ and the required result holds.

In the notation of Lemma 2.3, Seymour [18, (2.3)] proved that $\left\{x_{1}, x_{2}, \ldots, x_{m}\right\}$ contains a 2-cocircuit provided $M$ satisfies the additional condition that $M \backslash x_{m}$ is not 2-connected. Earlier he [17, (2.2)], Murty [10, Lemma 3.1] and White [24, Lemma 4.6] had independently shown that a minimally 2-connected matroid with at least two elements has a 2-cocircuit.

The next theorem, an alternative strengthening of Seymour's result, underlies most of the remaining results in this section.

(2.4) THEOREM. Let $C$ be a circuit of a 2-connected matroid $M$ such that for all $x$ in $C, M \backslash x$ is not 2-connected. Then either $M$ is the circuit $C$ or $C$ contains at least two distinct nontrivial series classes of $M$.

Proof. We argue by induction on $|C|$. If $|C|=1$, then the result is trivially true. Now suppose that $|C| \geqslant 2$. Then, by Lemma 2.3, $M$ has a 2-cocircuit $\{x, y\}$ meeting $C$. If every element of $C$ is in series with $x$, then it follows, since $M$ is 2-connected, that $C=E(M)$; for, if there is an element $e$ in $E(M) \backslash C$, then $M$ has a circuit $C^{\prime}$ containing $e$ and meeting $C$. But, as a circuit and a cocircuit of $M$ cannot have exactly one common element, we obtain the contradiction that $C^{\prime} \supsetneq C$. It follows that we may suppose that $C$ contains an element $z$ which is not in series with $x$. Consider $M / z$. By Lemma 2.1, this matroid is 2-connected. Moreover, $C \backslash z$ is a circuit of $M / z$. Now, if $a$ is an element of $C \backslash z$, then consider $M / z \backslash a$. If this is 2-connected, then, as $M \backslash a$ is not 2-connected, it follows from Lemma 2.2 that $\{a, z\}$ is a 2-cocircuit of $M$. Evidently $\{a, z\} \subseteq C$, and as $z$ is not in series with $x$, the required result holds. Thus we may assume that $M / z \backslash a$ is not 2-connected for all $a$ in $C \backslash z$. The required result now follows on applying the induction assumption. 
The preceding result is best-possible for if we take the cycle matroid of the plane graph $G$ shown in Figure 1, the cycle $C$ bounding the exterior face of $G$ contains only two distinct nontrivial series classes. It is not difficult to extend this to give examples of matroids in which $|C|$ is arbitrarily large and yet $C$ still contains only two nontrivial series classes.

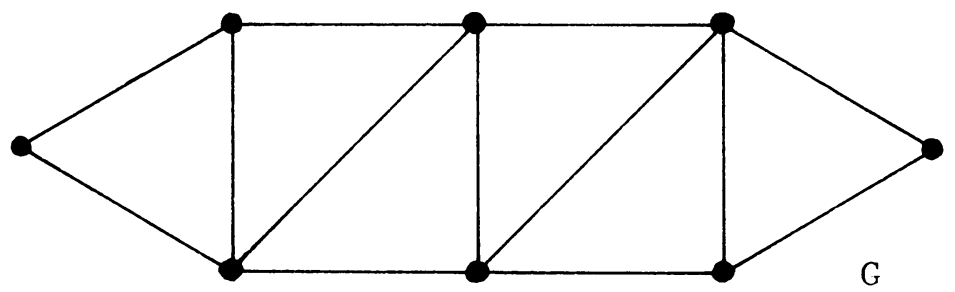

Figure 1

The next result, which generalizes a graph-theoretic result of Dirac [5, Proposition 3] and Plummer [16, Corollary 1b], is an immediate consequence of the preceding theorem.

(2.5) COROLlaRY. If a 2-connected matroid $M$ has a 3-circuit $T$ such that for all $x$ in $T$, the matroid $M \backslash x$ is not 2-connected, then $M$ is the 3-circuit $T$.

(2.6) Corollary. Let $M$ be a 2-connected matroid other than a single circuit. Suppose that $A \subseteq E(M)$ such that for all $a$ in $A, M \backslash a$ is not 2-connected. Then either $A$ is independent or $A$ contains at least $|A|-\operatorname{rk} A+1$ nontrivial series classes of $M$.

Proof. Suppose that $A$ is dependent and let $A^{\prime}$ be the union of all circuits of $M$ contained in $A$. We shall prove that $A^{\prime}$ contains at least $\left|A^{\prime}\right|-$ rk $A^{\prime}+1$ nontrivial series classes of $M$. The required result will then follow since $|A|-\operatorname{rk} A=\left|A^{\prime}\right|-$ rk $A^{\prime}$. As a circuit and a cocircuit of a matroid cannot have exactly one common element, if $C^{*}$ is a 2-cocircuit of $M$ meeting $A^{\prime}$, then $C^{*} \subseteq A^{\prime}$, and moreover, $C^{*}$ is a 2-cocircuit of $M \mid A^{\prime}$. Now let $X$ be the set of elements of $A^{\prime}$ which are contained in a 2-cocircuit of $M$. Then, by Theorem $2.4, X$ meets every circuit of $M \mid A^{\prime}$. Since the cobases of a matroid are the minimal sets meeting every circuit, it follows that $X$ contains a cobase $B^{*}$ of $M \mid A^{\prime}$. Choose an element $b$ of $B^{*}$ and let $C_{b}$ be the fundamental circuit of $b$ in $M \mid A^{\prime}$ with respect to $A^{\prime} \backslash B^{*}$. By the choice of $B^{*}, M$ has a 2-cocircuit, say $\{b, c\}$, containing $b$. Moreover, by Theorem $2.4, C_{b}$ contains another 2-cocircuit $\{d, e\}$ which is contained in a different series class from $\{b, c\}$. Now let $B^{*}=\left\{x_{1}, x_{2}, \ldots, x_{m}\right\}$ where $x_{1}=b$, and let $\left\{x_{1}, y_{1}\right\}$, $\left\{x_{2}, y_{2}\right\}, \ldots,\left\{x_{m}, y_{m}\right\}$ be 2-cocircuits of $M$ where $y_{1}=c$. We complete the proof by showing that $\left\{x_{1}, y_{1}\right\},\left\{x_{2}, y_{2}\right\}, \ldots,\left\{x_{m}, y_{m}\right\},\{d, e\}$ are contained in different series classes of $M$. Since $B^{*}$ is a cobase of $M \mid A^{\prime}$, it contains at most one element of each series class of $M$ and therefore $\left\{x_{1}, y_{1}\right\},\left\{x_{2}, y_{2}\right\}, \ldots,\left\{x_{m}, y_{m}\right\}$ are contained in distinct nontrivial series classes of $M$. It remains to show that none of these classes contains $\{d, e\}$. But, if for some $i,\left\{d, e, x_{i}, y_{i}\right\}$ is contained in a series class of $M$, then this series class is contained in $C_{b}$. It follows that $i=1$, hence 
$\left\{x_{i}, y_{i}\right\}=\{b, c\}$ and we have a contradiction to the fact that $\{b, c\}$ and $\{d, e\}$ are contained in different series classes of $\boldsymbol{M}$.

The next result is an immediate consequence of the preceding corollary, slightly strengthens [14, Proposition 4.13].

(2.7) Corollary. Let $M$ be a minimally 2-connected matroid which is not a circuit. Then $M$ has at least cork $M+1$ nontrivial series classes and therefore has at least cork $M+1$ pairwise disjoint 2-cocircuits.

We now come to apply Theorem 2.4 to graphs. First, however, we note the following result of Mader.

(2.8) TheOREM [8, SATZ 1]. Let $C$ be a cycle of a simple $n$-connected graph $G$ such that for all edges $x$ of $C, G \backslash x$ is not $n$-connected. Then $C$ meets a vertex which has degree $n$ in $G$.

In the case $n=2$, we can strengthen this result as follows.

(2.9) TheOREM. Let $G$ be a 2-connected graph without loops and $C$ be a cycle of $G$ such that for all edges $x$ of $C$, the graph $G \backslash x$ is not 2-connected. Then either $G$ is the cycle $C$ or $C$ meets two vertices of $G$ of degree two which are separated on $C$ by vertices of degree greater than two.

Proof. As $G$ is 2-connected, $|V(G)| \geqslant 3$ and therefore, by (1.3), $M(G)$ is 2 -connected. The required result will follow if we can show that either $G$ is a cycle or $C$ contains at least two nontrivial series classes of $M(G)$ each of which contains a vertex 2-cocircuit of $G$. We shall show this by induction on $|C|$ noting that the result holds for $|C| \leqslant 3$ (see Corollary 2.5). Now assume that $G$ is not a cycle. Then, by Theorem 2.4, $C$ contains at least two nontrivial series classes, $A_{1}$ and $A_{2}$ say. We may assume that $A_{1}$ does not contain a vertex 2-cocircuit of $M(G)$. Let $\{a, b\}$ be a 2-cocircuit contained in $A_{1}$. Then $G \backslash\{a, b\}$ has precisely two components, $H_{1}$ and $H_{2}$ say. Let $a=\left(a_{1}, a_{2}\right)$ and $b=\left(b_{1}, b_{2}\right)$. Then clearly $a_{1}, a_{2}, b_{1}$ and $b_{2}$ are distinct. For $i=1,2$, assume that $a_{i}, b_{i} \in H_{i}$, and form $G_{i}$ as follows. Let $V\left(G_{i}\right)=V\left(H_{i}\right) \cup\{i\}$ and $E\left(G_{i}\right)=E\left(H_{i}\right) \cup\left\{\left(a_{i}, i\right),\left(b_{i}, i\right)\right\}$. Then clearly $G_{1}$ and $G_{2}$ are both 2-connected. Moreover, neither $G_{1}$ nor $G_{2}$ is a cycle as otherwise $A_{1}$ contains a vertex 2 -cocircuit of $G$. Now for $i=1,2$, let

$$
C_{i}=\left(C \cap E\left(H_{i}\right)\right) \cup\left\{\left(a_{i}, i\right),\left(b_{i}, i\right)\right\} .
$$

Then evidently $C_{i}$ is a cycle of $G_{i}$. In addition, it is not difficult to check that for all $x$ in $C_{i}$, the graph $G_{i} \backslash x$ is not 2-connected. Then, as $\left|C_{i}\right|<|C|$, it follows by the induction assumption that $C_{i}$ contains at least two nontrivial series classes $A_{i, 1}$ and $A_{i, 2}$ of $M\left(G_{i}\right)$ each of which contains a vertex 2-cocircuit of $G_{i}$. Assume without loss of generality that $\left\{\left(a_{i}, i\right),\left(b_{i}, i\right)\right\} \subseteq A_{i, 2}$. Then it is straightforward to show that $A_{1,1}$ and $A_{2,1}$ are distinct nontrivial series classes of $M(G)$ each containing a vertex 2-cocircuit of $M(G)$. Thus by induction the required result is established.

If one strengthens the hypothesis of the preceding result by insisting that the graph $G$ be minimally 2-connected, then one obtains a result of Dirac [5, Theorem 5] and Plummer [16, Corollary 2a]. The next three results follow from Theorem 2.9 
in the same way that Corollaries 2.6 and 2.7 follow from Theorem 2.4. For a graph $G$, the number of connected components of $G$ will be denoted by $k(G)$.

(2.10) Corollary. Let $G$ be a 2-connected loopless graph other than a cycle. Suppose that $A \subseteq E(G)$ such that for all $a$ in $A, G \backslash a$ is not 2-connected. Then either $A$ is the edge-set of a forest, or $V(A)$ contains at least $|A|-|V(A)|+k(A)+1$ pairwise nonadjacent vertices having degree two in $G$.

(2.11) Corollary. Let $G$ be a minimally 2-connected graph other than a cycle. Then there is a set $U$ containing at least $|E(G)|-|V(G)|+2$ vertices such that each member of $U$ has degree two and every path in $G$ joining two members of $U$ contains $a$ vertex of degree greater than two.

(2.12) COROLlary. A minimally 2-connected graph $G$ having at least four edges has at least $|E(G)|-|V(G)|+2$ pairwise nonadjacent vertices of degree two.

The last result can also be deduced from the results of Dirac [5, Theorem 5] and Plummer [16, Corollary 2a] which Theorem 2.9 extends. We now compare the bound in Corollary 2.12 with the following bound which comes from a result of Dirac [5, (6), (5)], noting that the last result can also be used to give an alternative derivation of Corollary 2.12.

(2.13) Theorem [5, (6), (5)]. A minimally 2-connected graph $G$ has at least $(|V(G)|+4) / 3$ vertices of degree two.

The number of vertices of degree $k$ in a graph $G$ will be denoted by $\nu_{k}$.

(2.14) Proposition. Let $G$ be a minimally 2-connected graph having at least four edges. Then

$$
\nu_{2} \geqslant\left\{\begin{array}{l}
\frac{|V(G)|+5}{3} \text { for }|V(G)| \leqslant|E(G)|<\frac{4|V(G)|-2}{3}, \\
|E(G)|-|V(G)|+2 \text { for } \frac{4|V(G)|-2}{3}<|E(G)|<2|V(G)|-4 .
\end{array}\right.
$$

Proof. Dirac [5, (7)] has shown that $E(G)$ contains at least $|V(G)|$ and at most $2|V(G)|-4$ edges. By Corollary 2.12 and Theorem 2.13,

$$
\nu_{2} \geqslant \max \{(|V(G)|+4) / 3,|E(G)|-|V(G)|+2\},
$$

and it is straightforward to check that $|E(G)|-|V(G)|+2>(|V(G)|+4) / 3$ if and only if $|E(G)| \geqslant(4|V(G)|-2) / 3$. Thus

$$
\nu_{2} \geqslant\left\{\begin{array}{l}
\frac{|V(G)|+4}{3} \text { for }|V(G)| \leqslant|E(G)|<\frac{4|V(G)|-2}{3}, \\
|E(G)|-|V(G)|+2 \text { for } \frac{4|V(G)|-2}{3}<|E(G)|<2|V(G)|-4 .
\end{array}\right.
$$

But if $|E(G)|<(4|V(G)|-2) / 3$, then

$$
2|E(G)|<(8|V(G)|-4) / 3 \text {. }
$$

Now, by summing the vertex degrees of $G$ we get

$$
2|E(G)| \geqslant 2 \nu_{2}+3\left(|V(G)|-\nu_{2}\right)
$$


On combining (2.15) and (2.16), we get $(8|V(G)|-4) / 3>3|V(G)|-\nu_{2}$. Hence $\nu_{2}>(|V(G)|+4) / 3$, and therefore, $\nu_{2} \geqslant(|V(G)|+5) / 3$.

The graph $K_{2,3}$ is one of infinitely many graphs which show that in Theorem 2.13 we cannot replace $(|V(G)|+4) / 3$ by $(|V(G)|+5) / 3$ (see [1, pp. 15-16 and p. 48, Exercise 20]), although clearly this replacement can be made unless $|V(G)| \equiv 2$ (mod 3). The structure of those minimally 2-connected graphs $G$ having exactly $\{(|V(G)|+4) / 3\}$ vertices of degree two will be determined elsewhere.

It is natural to ask whether Proposition 2.14 can be extended to minimally $n$-connected graphs for $n \geqslant 3$. The proof of Corollary 2.12 relied heavily on the fact that in a minimally 2-connected graph with at least four edges, each cycle meets at least two nonadjacent vertices of degree two. The corresponding, but slightly weaker, result for minimally $n$-connected graphs is an immediate consequence of Theorem 2.8. Mader [8, Satz 2] used this result to show that in a minimally $n$-connected graph $G$,

$$
\nu_{n} \geqslant \frac{(n-1)|V(G)|+2}{2 n-1} .
$$

Recently, Mader [9, Theorem 17] has strengthened (2.17) to get that

$$
\nu_{n} \geqslant \frac{(n-1)|V(G)|+2 n}{2 n-1} .
$$

The following application of Theorem 2.8, although straightforward, seems new, yet the bound it gives frequently sharpens (2.18). The proof is similar to the proof of Corollary 2.6.

(2.19) Proposition. Let $G$ be a minimally $n$-connected graph where $n>2$. Then

$$
\nu_{n} \geqslant \frac{|E(G)|-|V(G)|+1}{n-1} .
$$

Proof. Let $X$ be the set of edges of $G$ which are incident with some vertex of degree $n$. Then, by Theorem $2.8, X$ meets every cycle of $G$. That is, $X$ meets every circuit of $M(G)$, and thus $X$ contains a cobase $B^{*}$ of $M(G)$. Now the set of edges incident with a vertex $v$ of $G$ is a cocircuit of $M(G)$, so $B^{*}$ contains at most $n-1$ of the edges of a vertex $n$-cocircuit of $G$. Hence $\nu_{n}>\left|B^{*}\right| /(n-1)$. As $\left|B^{*}\right|=$ $|E(G)|-|V(G)|+1$, the required result follows.

In the case $n=3$, (2.18) was first proved by Halin [7, Satz 6]. His proof was based on the fact that a cycle in a minimally 3-connected graph meets at least two vertices of degree three [7, Satz 5]. Using this, the proof method of Propositions 2.14 and 2.19 can now be extended to give the following analogue of Proposition 2.14 .

(2.20) Proposition. Let $G$ be a minimally 3-connected graph. Then

$$
\nu_{3} \geqslant\left\{\begin{array}{l}
\frac{2|V(G)|+7}{5} \text { for } \frac{3|V(G)|}{2} \leqslant|E(G)|<\frac{9|V(G)|-3}{5} \\
\frac{|E(G)|-|V(G)|+3}{2} \text { for } \frac{9|V(G)|-3}{5}<|E(G)|<3|V(G)|-6 .
\end{array}\right.
$$


Proof. As every vertex of $G$ has degree at least three, $|E(G)|>3|V(G)| / 2$. Moreover, $|E(G)| \leqslant 3|V(G)|-6$ (see, for example, [14, Theorem 4.7]). We shall now show that $\nu_{3} \geqslant \frac{1}{2}(|E(G)|-|V(G)|+3)$. The rest of the proof then follows by generalizing the proof of Proposition 2.14. Let $X$ and $B^{*}$ be as in the proof of Proposition 2.19. Then we need to show that $\nu_{3} \geqslant \frac{1}{2}\left(\left|B^{*}\right|+2\right)$. Choose an element $a$ of $B^{*}$. Then $a$ is in a vertex 3-cocircuit $C_{1}^{*}$. Now let $C_{a}$ be the fundamental circuit of $a$ with respect to $E(G) \backslash B^{*}$. Then $C_{a}$ meets a vertex 3-cocircuit $C_{2}^{*}$ which is different from $C_{1}^{*}$. Since $\left|C_{i}^{*} \cap C_{a}\right| \geqslant 2$ for $i=1,2,\left|B^{*} \cap\left(C_{1}^{*} \cup C_{2}^{*}\right)\right| \leqslant 3$. Therefore, as $B^{*}$ contains at most two elements of any vertex 3-cocircuit, $\nu_{3}$ $\geqslant \frac{1}{2}\left(\left|B^{*} \backslash\left(C_{1}^{*} \cup C_{2}^{*}\right)\right|\right)+2$. That is, $\nu_{3} \geqslant \frac{1}{2}\left(\left|B^{*}\right|+1\right)$ with equality holding only if $\left|B^{*} \cap C_{1}^{*}\right|=2$ and $\left|B^{*} \cap\left(C_{1}^{*} \cup C_{2}^{*}\right)\right|=3$. Thus we may assume that there is an element $b$ in $\left(B^{*} \cap C_{2}^{*}\right) \backslash C_{1}^{*}$, for otherwise the required result holds. Now let $C_{b}$ be the fundamental circuit of $b$ with respect to $E(G) \backslash B^{*}$. Then $C_{b}$ meets a vertex 3-cocircuit $C_{3}^{*}$ different from $C_{2}^{*}$. Moreover, since $b \notin C_{1}^{*}$, it follows that $C_{3}^{*} \neq C_{1}^{*}$. Now $\left|B^{*} \backslash\left(C_{1}^{*} \cup C_{2}^{*} \cup C_{3}^{*}\right)\right| \geqslant\left|B^{*}\right|-4$, so $\nu_{3} \geqslant \frac{1}{2}\left(\left|B^{*}\right|-4\right)+3$; that is, $\nu_{3}$ $\geqslant \frac{1}{2}\left(\left|B^{*}\right|+2\right)$, as required.

A natural question now is what can be said when $n \geqslant 3$ about the occurrence of $n$-cocircuits in minimally $n$-connected matroids. Some progress has been made in this direction for $n=3$ and $n=4$ [14], [25].

(2.21) Theorem [14, TheOrem 2.5]. If $C$ is a circuit of a minimally 3-connected matroid $M$ and $|E(M)| \geqslant 4$, then $M$ has at least two distinct 3-cocircuits meeting $C$.

It is not difficult to find examples showing that this result is best-possible. Using it and the proof technique of Proposition 2.20, one can deduce that a minimally 3-connected matroid $M$ with at least four elements has at least $\frac{1}{2} \operatorname{cork} M+1$ 3-cocircuits. This result is also best-possible. Some partial results on the question of whether a minimally 4-connected matroid has a 4-cocircuit have been obtained by Wong [25].

The results earlier in this section generalize known results for minimally 2-connected matroids and graphs by weakening the requirement of minimal 2-connectedness. In this context one is led to ask whether the hypothesis of Theorem 2.21 can be weakened so that one does not require the matroid $M$ to be minimally 3-connected, but only that the deletion of elements of the circuit $C$ should destroy 3-connectedness. The corresponding problem for graphs, which if true would strengthen Theorem 2.8 in the case $n=3$, also seems to be open.

3. A characterization of minimally 2-connected matroids. The main result of this section solves a problem of Murty [10, p. 53] by providing a rather elementary yet useful characterization of minimally 2 -connected matroids. This characterization is then used to give short proofs of the main results of Murty's paper [10]. We shall use the operation of series connection of matroids and a number of properties of this operation proved by Brylawski [3]. Let $M_{1}$ and $M_{2}$ be matroids having disjoint ground sets $S_{1}$ and $S_{2}$ respectively. Choose an element $p_{1}$ from $S_{1}$ and an element $p_{2}$ from $S_{2}$. These elements are called the basepoints of $M_{1}$ and $M_{2}$ respectively. Let $p$ be a new element. Then the series connection $S\left(\left(M_{1} ; p_{1}\right),\left(M_{2} ; p_{2}\right)\right)$ of $M_{1}$ and $M_{2}$ 
with respect to the basepoint $p_{1}$ and $p_{2}$ is the matroid on $\left(S_{1} \backslash p_{1}\right) \cup\left(S_{2} \backslash p_{2}\right) \cup p$ whose circuits are the circuits of $M_{1}$ not containing $p_{1}$, the circuits of $M_{2}$ not containing $p_{2}$, and all sets of the form $\left(C_{1} \backslash p_{1}\right) \cup\left(C_{2} \backslash p_{2}\right) \cup p$ where $p_{1} \in C_{1}$, $p_{2} \in C_{2}$ and $C_{1}$ and $C_{2}$ are circuits of $M_{1}$ and $M_{2}$ respectively. If the basepoints are clear the series connection will sometimes be denoted $S\left(M_{1}, M_{2}\right)$. Series connection and the corresponding dual operation, parallel connection, were studied in detail by Brylawski [3]. More recently, Seymour [19] has looked at the closely related operation of 2-sum of matroids, the 2-sum of $M_{1}$ and $M_{2}$ being precisely $S\left(M_{1}, M_{2}\right) / p[22]$.

(3.1) TheOREM. A matroid $M$ is minimally 2-connected if and only if $|E(M)| \geqslant 3$, and either $M$ is 2-connected and every element of $M$ is in a 2-cocircuit, or $M=S\left(\left(M_{1} / q_{1} ; p_{1}\right),\left(M_{2} / q_{2} ; p_{2}\right)\right)$ where both $M_{1}$ and $M_{2}$ are minimally 2-connected having at least four elements and $\left\{p_{1}, q_{1}\right\}$ and $\left\{p_{2}, q_{2}\right\}$ are cocircuits of $M_{1}$ and $M_{2}$ respectively.

Proof. Evidently if $M$ is a 2-connected matroid for which $|E(M)| \geqslant 3$ and every element is in a 2-cocircuit, then $M$ is minimally 2-connected. Now if $M_{1}$ and $M_{2}$ are minimally 2-connected matroids each having at least three elements, then, by Theorem 2.4 , each certainly possesses a 2 -cocircuit. Consider $M=S\left(\left(M_{1} / q_{1} ; p_{1}\right)\right.$, $\left.\left(M_{2} / q_{2} ; p_{2}\right)\right)$ where $\left\{p_{1}, q_{1}\right\}$ and $\left\{p_{2}, q_{2}\right\}$ are cocircuits of $M_{1}$ and $M_{2}$ respectively. Then since, by Lemma $2.1, M_{1} / q_{1}$ and $M_{2} / q_{2}$ are 2-connected, it follows by [3, Proposition 4.6] that $M$ is 2-connected.

We now show that $M$ is minimally 2 -connected. Firstly, $M \backslash p=\left(M_{1} / q_{1}\right) \backslash p_{1} \oplus$ $\left(M_{2} / q_{2}\right) \backslash p_{2}$ [3, Proposition 4.9], so $M \backslash p$ is not 2-connected. Now suppose that $e \in E\left(M_{1} / q_{1}\right) \backslash p_{1}$ and consider $M \backslash e$. We have $M \backslash e=S\left(M_{1} / q_{1}, M_{2} / q_{2}\right) \backslash e=$ $S\left(M_{1} / q_{1} \backslash e, M_{2} / q_{2}\right)$ where the last step holds by [3, Proposition 4.7]. If $M_{1} / q_{1} \backslash e$ is not 2-connected, then by [3, Proposition 4.6], $M \backslash e$ is not 2-connected, as required. Thus suppose that $M_{1} / q_{1} \backslash e$ is 2-connected. Then by Lemma $2.2, q_{1}$ is a loop or a coloop of $M_{1} \backslash e$. But $M_{1}$ has no loops or coloops, so $\left\{e, q_{1}\right\}$ is a cocircuit of $M_{1}$. Therefore, as $\left\{p_{1}, q_{1}\right\}$ is a cocircuit, $\left\{e, p_{1}\right\}$ is also a cocircuit of $M_{1}$ and hence is a cocircuit of $M_{1} / q_{1}$. Thus $p_{1}$ is a coloop of $M_{1} / q_{1} \backslash e$ and thus, by [3, Proposition 4.5], $M \backslash e$ is not 2-connected. It follows that $M$ is minimally 2-connected, as required.

For the converse, suppose that $M$ has an element $p$ which is not in a 2-cocircuit. Now $M \backslash p$ is not 2-connected and since $M$ has no 2-cocircuits containing $p$, the matroid $M \backslash p$ has no component with fewer than two elements. Let $M \backslash p=N_{1}$ $\oplus N_{2}$. Then, by [3, Proposition 4.10], $M=S\left(M / E\left(N_{1}\right), M / E\left(N_{2}\right)\right)$ where $p$ is the basepoint of both $M / E\left(N_{1}\right)$ and $M / E\left(N_{2}\right)$. Let $N_{3}=M / E\left(N_{1}\right)$ and $N_{4}=$ $M / E\left(N_{2}\right)$. Then each of $N_{3}$ and $N_{4}$ has at least three elements. Now, as $M=$ $S\left(N_{3}, N_{4}\right)$ and $M$ is 2-connected, by [3, Proposition 4.6] again, each of $N_{3}$ and $N_{4}$ is also 2-connected. If $e \in E(M)$ and $e \neq p$, then $e \in E\left(N_{3}\right) \backslash p$ or $e \in E\left(N_{4}\right) \backslash p$, so suppose the former. Then as $M \backslash e=S\left(N_{3} \backslash e, N_{4}\right)$ and $M \backslash e$ is not 2-connected, $N_{3} \backslash e$ is not 2-connected. Thus for all elements $e$ of $N_{3}$, except possibly $p$, the matroid $N_{3} \backslash e$ is not 2-connected. Similarly, $N_{4} \backslash f$ is not 2-connected for all 
elements $f$ of $N_{4}$ except possibly $p$. Now for $i=1,2$, add an element $q_{i}$ in series with $p$ in $N_{i+2}$ to get a new matroid $M_{i}$ which is clearly 2-connected. In fact, it is not difficult to check that $M_{i}$ is minimally 2-connected. Then $M$ can be obtained by contracting $q_{1}$ and $q_{2}$ from $M_{1}$ and $M_{2}$ respectively and then taking the series connection of $M_{1} / q_{1}$ and $M_{2} / q_{2}$ with respect to the basepoint $p$. Finally, as each of $N_{3}$ and $N_{4}$ has at least three elements, each of $M_{1}$ and $M_{2}$ has at least four elements.

(3.2) Corollary [10, Theorems 3.2 AND 3.4]. If $r \geqslant 3$, a minimally 2-connected matroid $M$ of rank $r$ has at most $2 r-2$ elements, the upper bound being attained if and only if $M \cong M\left(K_{2, r-1}\right)$.

Proof. We argue by induction on $|E(M)|$. If every element of $M$ is in a 2-cocircuit, then consider $M^{*}$. Deleting a single element from every parallel class of $M^{*}$ leaves a 2-connected matroid $N$ having the same rank as $M^{*}$. Evidently $N$ has at least rk $M^{*}+1$ elements with equality being attained only if $N$ is a circuit. Thus $\left|E\left(M^{*}\right)\right|=|E(M)| \geqslant 2\left(\right.$ rk $\left.M^{*}+1\right)=2(|E(M)|-r+1)$, and therefore $|E(M)|<$ $2(r-1)$ with equality being attained only if $M^{*} \cong C_{r-1}^{2}$, an $(r-1)$-circuit in which each element has been replaced by a pair of parallel elements. But if $M^{*} \cong C_{r-1}^{2}$, then $M \cong M\left(K_{2, r-1}\right)$.

We may now suppose that $M$ has an element $p$ which is not in a 2-cocircuit. Then, by Theorem $3.1, M=S\left(\left(M_{1} / q_{1} ; p_{1}\right),\left(M_{2} / q_{2} ; p_{2}\right)\right)$ where, for $i=1,2$, $\left\{p_{i}, q_{i}\right\}$ is a cocircuit of $M_{i}$, and $M_{i}$ is minimally 2-connected having at least four elements and hence having rank at least three. Now, by [3, Theorem 6.16(i)], rk $M=\operatorname{rk}\left(M_{1} / q_{1}\right)+\operatorname{rk}\left(M_{2} / q_{2}\right)$ and thus

$$
\text { rk } M=\text { rk } M_{1}+\text { rk } M_{2}-2 \text {. }
$$

Moreover,

$$
|E(M)|=\left|E\left(M_{1}\right)\right|+\left|E\left(M_{2}\right)\right|-3,
$$

and, by the induction assumption, $\left|E\left(M_{i}\right)\right| \leqslant 2 \mathrm{rk} M_{i}-2$ for $i=1,2$. Thus $\left|E\left(M_{1}\right)\right|+\left|E\left(M_{2}\right)\right| \leqslant 2$ (rk $M_{1}+$ rk $M_{2}-2$ ), and, by (3.3) and (3.4), $|E(M)|<2 r$ - 3. Hence, by induction, the required result is proved.

Theorem 3.1 may also be used to give alternative proofs of several results for minimally 2-connected matroids such as Corollary 2.7 .

4. Colouring. The chromatic number of a loopless graph is the least positive integer at which the value of its chromatic polynomial is positive. For loopless matroids in general there are some difficulties in defining the chromatic number (see [23, p. 264]). However, such problems do not arise for regular matroids. Thus if $M$ is a loopless regular matroid having chromatic polynomial $P(M ; \lambda)$ (see, for example, [23, p. 262]) its chromatic number $\chi(M)$ is $\min \left\{j \in \mathbf{Z}^{+}: P(M ; j)>0\right\}$. It can be shown (see, for example, [11, p. 17]) that, as for graphs, $P(M ; k)>0$ for all integers $k$ such that $k \geqslant \chi(M)$.

For a loopless matroid $M$ representable over a finite field $G F(q)$, Crapo and Rota [4, Chapter 16] introduced an important invariant which, when $M$ is regular, is closely related to its chromatic number. The critical exponent $c(M ; q)$ of $M$ is 
$\min \left\{j \in \mathbf{Z}^{+}: P\left(M ; q^{j}\right)>0\right\}$. It follows from [4, p. 16.4] that $P\left(M ; q^{k}\right)>0$ for all positive integers $k$ and moreover, $P\left(M ; q^{k}\right)>0$ if $k>c(M ; q)$.

The main result of this section is the following.

(4.1) THEOREM. Let $M$ be a minimally $n$-connected matroid where $n=2$ or 3 . If $M$ is representable over $G F(q)$, then $c(M ; q) \leqslant 2$ for $q \leqslant n$ and $c(M ; q)=1$ for $q>n$. Moreover, if $M$ is regular, then $\chi(M) \leqslant n+1$.

The proof of this will use three lemmas.

(4.2) LEMMA. Let $M$ be a minimally $n$-connected matroid where $n=2$ or 3 and suppose $T \subseteq E(M)$. Then $M \mid T$ has a cocircuit having at most $n$ elements.

Proof. If $T$ does not contain a circuit of $M$, then $M \mid T$ is free, so $M \mid T$ has a coloop. If $T$ does contain a circuit $C$ of $M$, then, by Theorems 2.4 and $2.21, C$ meets an $n$-cocircuit $C^{*}$ of $M$. Now $C^{*}$ certainly contains a cocircuit of $M \mid T$ and the required result follows.

(4.3) LeMMA [12, LeMMA 5]. If $M$ is a matroid representable over $G F(q)$ and $M$ is minimal having critical exponent $k+1$, then every cocircuit of $M$ has at least $q^{k}$ elements.

The analogue of the preceding result for regular matroids is as follows.

(4.4) LEMMA [13, THeOREM 3]. If $M$ is a regular matroid which is minimal having chromatic number $k+1$, then every cocircuit of $M$ has at least $k$ elements.

Proof of Theorem 4.1. We shall prove the result for $M$ representable over $G F(q)$ by using Lemmas 4.2 and 4.3. The result for $M$ regular follows similarly by using Lemma 4.4 in place of Lemma 4.3 .

Suppose $c(M ; q)=k+1$. Then by deleting elements from $M$, we obtain a restriction $N$ which is minimal having critical exponent $k+1$. By Lemma 4.3, every cocircuit of $N$ has at least $q^{k}$ elements. But, by Lemma 4.2, $N$ has a cocircuit having at most $n$ elements. Thus $n \geqslant q^{k}$ and the required result follows.

\section{REFERENCES}

1. B. Bollobás, Extremal graph theory, London Math. Soc. Mono., no. 11, Academic Press, London, New York and San Francisco, 1978.

2. J. A. Bondy and U. S. R. Murty, Graph theory with applications, Macmillan, London; American Elsevier, New York, 1976.

3. T. H. Brylawski, A combinatorial model for series-parallel networks, Trans. Amer. Math. Soc. 154 (1971), 1-22.

4. H. H. Crapo and G.-C. Rota, On the foundations of combinatorial theory: combinatorial geometries, Preliminary ed., MIT Press, Cambridge, Mass. and London, 1970.

5. G. A. Dirac, Minimally 2-connected graphs, J. Reine Angew. Math. 228 (1967), 204-216.

6. J. E. Graver and M. E. Watkins, Combinatorics with emphasis on the theory of graphs, SpringerVerlag, New York, Heidelberg and Berlin, 1977.

7. R. Halin, Untersuchungen über minimale n-fach zusammenhängende Graphen, Math. Ann. 182 (1969), 175-188.

8. W. Mader, Ecken vom Grad $n$ in minimalen $n$-fach zusammenhängenden Graphen, Arch. Math. (Basel) 23 (1972), 219-224.

9. Connectivity and edge-connectivity in finite graphs, Surveys in Combinatorics (B. Bollobás, ed.), Cambridge Univ. Press, New York, 1979, pp. 66-95. 
10. U. S. R. Murty, Extremal critically connected matroids, Discrete Math. 8 (1974), 49-58.

11. J. G. Oxley, Colouring, packing and the critical problem, Quart. J. Math. Oxford Ser. (2) 29 (1978), 11-22.

12. On a covering problem of Mullin and Stanton for binary matroids, Aequationes Math. 20 (1980), 104-112.

13. __ A generalization of a covering problem of Mullin and Stanton for matroids, Proc. Sixth Austral. Combinatorics Conf., Lecture Notes in Math., vol. 748, Springer-Verlag, Berlin, Heidelberg and New York, 1979, pp. 92-97.

14. __ On matroid connectivity, Quart. J. Math. Oxford Ser. (2) (to appear).

15. __ On 3-connected matroids, Canad. J. Math. (to appear).

16. M. D. Plummer, On minimal blocks, Trans. Amer. Math. Soc. 134 (1968), 85-94.

17. P. D. Seymour, Matroid representation over GF(3), J. Combin. Theory. Ser. B 26 (1979), 159-173.

18. __ Packing and covering with matroid circuits, J. Combin. Theory Ser. B 28 (1980), 237-242.

19. __ Decomposition of regular matroids, J. Combin. Theory Ser. B 28 (1980), 305-359.

20. W. T. Tutte, Connectivity in matroids, Canad. J. Math. 18 (1966), 1301-1324.

21. __, Wheels and whirls, Lecture Notes in Math., vol. 211, Springer-Verlag, Berlin, Heidelberg and New York, 1971, pp. 1-4.

22. P. N. Walton and D. J. A. Welsh, On the chromatic number of binary matroids, Mathematika 27 (1980), 1-9.

23. D. J. A. Welsh, Matroid theory, London Math. Soc. Mono., no. 8, Academic Press, London, New York and San Francisco, 1976.

24. N. L. White, The bracket ring of a combinatorial geometry. II: Unimodular geometries, Trans. Amer. Math. Soc. 214 (1975), 233-248.

25. P.-K. Wong, On certain n-connected matroids, J. Reine Angew. Math. 299/300 (1978), 1-6.

Department of Mathematics, IAS, Australian National University, Canberra, Australia 